\title{
Images of Fractional Brownian motion with deterministic drift: Positive Lebesgue measure and non-empty interior
}

\author{
MOHAMED ERRAOUI \\ Department of mathematics, Faculty of science El jadida, \\ Chouaïb Doukkali University, Morocco \\ e-mail: erraoui@uca.ac.ma \\ YOUSSEF HAKIKI 1 \\ Department of mathematics, Faculty of science Semlalia, \\ Cadi Ayyad University, 2390 Marrakesh, Morocco \\ e-mail: youssef.hakiki@ced.uca.ma
}

\begin{abstract}
Let $B^{H}$ be a fractional Brownian motion in $\mathbb{R}^{d}$ of Hurst index $H \in(0,1), f:[0,1] \longrightarrow \mathbb{R}^{d}$ a Borel function and $A \subset[0,1]$ a Borel set. We provide sufficient conditions for the image $\left(B^{H}+f\right)(A)$ to have a positive Lebesgue measure or to have a non-empty interior. This is done through the study of the properties of the density of the occupation measure of $\left(B^{H}+f\right)$. Precisely, we prove that if the parabolic Hausdorff dimension of the graph of $f$ is greater than $H d$, then the density is a square integrable function. If, on the other hand, the Hausdorff dimension of $A$ is greater than $H d$, then it even admits a continuous version. This allows us to establish the result already cited.
\end{abstract}

Keywords: Fractional Brownian motion, Hausdorff dimension, Parabolic dimension.

Mathematics Subject Classification Primary 60J65

\section{Introduction}

Many sets from the trajectories of the fractional Brownian motion are somehow random fractals. These sets have an interesting geometric structure at all scales. Among the most studied in the literature, it is worth mentioning images and graphs of a fractional Brownian motion. For standard reference we refer to Adler [1], Kahane [10]. So, we start by recalling the exact Hausdorff dimension of the image $B^{H}(A)$ where $B^{H}$ is a fractional Brownian motion in $\mathbb{R}^{d}$ of Hurst index $H \in(0,1)$ and $A \subset \mathbb{R}$ is a Borel set. It is visibly that $B^{H}(A)$ is a random set in $\mathbb{R}^{d}$. There has been considerable interest in the study of its geometric and arithmetic properties. It is proved that almost surely

$$
\operatorname{dim} B^{H}(A)=\min \left\{\frac{\operatorname{dim}(A)}{H}, d\right\} .
$$

We write $\operatorname{dim}($.$) for the Hausdorff dimension, and refer to [6] and [10] for its definition and basic properties.$ Several works have focused strongly on determining whether $B^{H}(A)$ is a.s. a set of positive Lebesgue

\footnotetext{
${ }^{1}$ Supported by National Center for Scientific and Technological Research (CNRST)
} 
measure or a Salem set by comparing $\operatorname{dim}(A)$ and $H d$, see [10] and [18]. Two distinct cases are clearly apparent : $\operatorname{dim}(A)>H d$ or $\operatorname{dim}(A)<H d$.

In the first case, a proof based on Fourier transform due to Kahane (see [10]) provided a preliminary result, namely $B^{H}(A)$ a.s. has positive $d$-dimensional Lebesgue measure. Pitt [17] and Kahane [10] strengthened this result by proving that $B^{H}(A)$ a.s. has non-empty interior. However, it should be noted that, for the one dimensional Brownian motion the fact that $B^{H}(A)$ has non-empty interior when $\operatorname{dim}(A)>1 / 2$ is due to Kaufman in [11]. In the case $\operatorname{dim}(A)<H d$, Kahane [10] established that $B^{H}(A)$ is a.s. a Salem set. The reader might refer to the book of Kahane [10] for the precise definition and the basic properties of Salem set since it will not be treated here.

Recently Peres and Sousi [16] studied fractal properties of images and graphs of $B^{H}+f$ where $f$ : $[0,1] \rightarrow \mathbb{R}^{d}$ is a Borel measurable function. They expressed, for a Borel set $A \subset[0,1]$, the dimension of the image set $\left(B^{H}+f\right)(A)$ in terms of the so-called parabolic Hausdorff dimension of the graph of $f$ restricted to $A$ denoted by $\operatorname{dim}_{\Psi, H}\left(G r_{A}(f)\right)$, see Definition 2.1. Precisely, the authors stated that almost surely

$$
\operatorname{dim}\left(\left(B^{H}+f\right)(A)\right)=\min \left(\frac{\operatorname{dim}_{\Psi, H}\left(G r_{A}(f)\right)}{H}, d\right) .
$$

It is important to note that the parabolic Hausdorff dimension has first been considered by Taylor and Watson [19] for the study of polar sets of the heat equation. It has also been shown to be useful for analyzing the geometry of the images of Brownian motion, see Khoshnevisan and Xiao [13. Once again when $B^{H}$ is the multifractional Brownian motion $(\mathrm{mBm})$, the parabolic Hausdorff dimension again happens to be the relevant tool to describe the geometry of its graph and the images of sets, cf [3]. For all these reasons, it is therefore quite natural to ask the following question: what are the properties of the set $\left(B^{H}+f\right)(A)$ that can be explored deeply from the informations provided by the parabolic dimension of the graph of $f$ ? This issue will be the main goal of this work, which we consider as a continuation of [16].

In Section 2, first we give a comparison result for parabolic Hausdorff dimensions for different parameters. We look also seek to determine the exact value of $\operatorname{dim}_{\Psi, H}\left(G r_{A}(f)\right)$. It is already becoming apparent that calculation of parabolic Hausdorff dimensions can be a little involved, even for simple sets. For this, as a first step we establish lower and upper bounds of $\operatorname{dim}_{\Psi, H}\left(G r_{A}(f)\right)$, that take into account the Hölder continuity and $\operatorname{dim}(A)$. We note that the lower bound of $\operatorname{dim}_{\Psi, H}\left(G r_{A}(f)\right)$ can be seen as a kind of the Mastrand projection theorem. Secondly, in order to give sharp bounds, we looked for functions with known graphs, such as the paths of Hölder's continuous stochastic process. Precisely, we have considered the paths of the fractional Brownian motion with a Hurst parameter less than $H$. For this class we were able to calculate the exact value of the desired parabolic Hausdorff dimension.

In this section 3, our investigation focuses on the fractional Brownian motion with drift $\left(B^{H}+f\right)$ and turned to the question of whether the image $\left(B^{H}+f\right)(A)$ admit a positive Lebesgue measure or have a non-empty interior. The question we are examining arises from the subject of the polar functions of the planar Brownian motion initiated by Graversen [9] and explored in a more general context, including both the spatial dimension and the Hölder property, by Le Gall [14] and Antunović et al [2]. Here the idea is to construct, under the condition $\operatorname{dim}_{\Psi, H}\left(G r_{A}(f)\right)>H d$, a probability $\nu$ carried by $A$ such that its image measure $\mu$ under the mapping $A \ni t \longrightarrow\left(B^{H}+f\right)(t)$, commonly known as occupation measure, which is supported by $\left(B^{H}+f\right)(A)$ has a density with respect to the Lebesgue measure. This latter density is known as occupation density and interpreted as local time over the set $A$. The idea of introducing local times as densities of occupation measure has been fruitful in a variety of contexts. Important papers in this direction are Geman and Horowitz [7] and Geman, Horowitz and Rosen [8]. Our first result indicates that image $\left(B^{H}+f\right)(A)$ admit a positive Lebesgue measure since the occupation density is a square integrable function. In terms of the second part of our question, we will follow the approach used in Kahane [10] and Pitt [17]. The keystone of this approach is to reduce the problem of covering an open set to the existence of a continuous occupation density function. To do this, we'll draw upon on the condition 
used in the case without drift, which is a little stronger than that already assumed, namely $\operatorname{dim}(A)>H d$, see Propositon 2.7. It should be noted that the results of this section are indeed extensions of those established by Pitt and Kahane for fractional Brownian motion to the case of fractional Brownian motion with variable drift through the comparison between $\operatorname{dim}_{\Psi, H}\left(G r_{A}(f)\right)$ and $H d$.

Our motivation for section 4 is as follows: in his note [14 on Graversen's conjecture on the polar functions for the planar Brownian motion, Le Gall asked if for each $\gamma<1 / d$ there exist $\gamma$-Hölder continuous functions which are non-polar for $d$-dimensional Brownian motion $B^{1 / 2}$. An answer is given by Antunović et al [2] in terms of modifications of the standard Hilbert curve perturbed by Brownian motion. Precisely, they showed that for any $\gamma<1 / d$, there exists a $\gamma$-Hölder continuous function $f:[0,1] \longrightarrow \mathbb{R}^{d}$ for which the set $\left(B^{1 / 2}-f\right)([0,1])$ covers an open set almost surely. On the other hand, as mentioned before when $\operatorname{dim}(A)<H d, B^{H}(A)$ is a.s. a Salem set. It is natural to ask whether for each $\alpha \in(0, \operatorname{dim}(A) / d)$ there exist $\alpha$-Hölder continuous function $f:[0,1] \rightarrow \mathbb{R}^{d}$ for which the range $\left(B^{H}+f\right)(A)$ has a non-empty interior a.s.?

Since $\operatorname{dim}(A)>\alpha d$ then the previous case allows us to assert that the set $\left(B^{\alpha}+f\right)(A)$ have a nonempty interior where $B^{\alpha}$ is another fractional Brownian motion with Hurst index possibly defined on different probability space. Our idea is to consider $B^{H}$ as a drift of $B^{\alpha}$. This induces us to bring them together on the same space while preserving their distributions. The best way to do this is to work on the product space and to consider processes on this latter.

Here are some notations that we will use throughout this work. $\|\cdot\|_{\infty}$ denotes the maximum norm on $\mathbb{R}^{d} \cdot\langle\cdot, \cdot\rangle$ and $\|\cdot\|$ are the ordinary scalar product and the Euclidean norm in $\mathbb{R}^{d}$ respectively. If $(E, \rho)$ is a metric space, then the Borel $\sigma$-algebra over $E$ will be denoted by $\mathcal{B}(E)$. We denote by $\operatorname{dim} A$ the Hausdorff dimension of a set $A \subset \mathbb{R}$. For a function $f: \mathbb{R}_{+} \rightarrow \mathbb{R}^{d}, G r_{A}(f)=\{(t, f(t)): t \in A\}$ is the graph of $f$ over the set $A$. We will use $C, C_{1}, \ldots, C_{4}$ to denote unspecified positive finite constants which may not necessarily be the same in each occurrence.

\section{Preliminaries on Parabolic Hausdorff dimension}

Let $B_{0}^{H}=\left\{B_{0}^{H}(t), t \geq 0\right\}$ be a real-valued fractional Brownian motion of Hurst index $H$ defined on a complete probability space $(\Omega, \mathcal{F}, \mathbb{P})$, i.e. a real valued Gaussian process with stationary increments and covariance function given by

$$
\mathbb{E}\left(B_{0}^{H}(s) B_{0}^{H}(t)\right)=\frac{1}{2}\left(|t|^{2 H}+|s|^{2 H}-|t-s|^{2 H}\right) .
$$

Let $B_{1}^{H}, \ldots, B_{d}^{H}$ be $d$ independent copies of $B_{0}^{H}$, then the stochastic process $B^{H}=\left\{B^{H}(t), t \geq 0\right\}$ given by

$$
B^{H}(t)=\left(B_{1}^{H}(t), \ldots ., B_{d}^{H}(t)\right),
$$

is called a $d$-dimensional fractional Brownian motion of Hurst index $H \in(0,1)$.

We start by giving the definition of the parabolic Hausdorff dimension.

Definition 2.1. Let $F \subset \mathbb{R}_{+} \times \mathbb{R}^{d}$ and $H \in(0,1)$. For all $\beta>0$ the $H$-parabolic $\beta$-dimensional Hausdorff content is defined by

$$
\Psi_{H}^{\beta}(F)=\inf \left\{\sum_{j} \delta_{j}^{\beta}: F \subseteq \bigcup_{j}\left[a_{j}, a_{j}+\delta_{j}\right] \times\left[b_{j, 1}, b_{j, 1}+\delta_{j}^{H}\right] \times \ldots \times\left[b_{j, d}, b_{j, d}+\delta_{j}^{H}\right]\right\}
$$

where the infimum is taken over all covers of $F$ by rectangles of the form given above. The $H$-parabolic Hausdorff dimension is then defined to be

$$
\operatorname{dim}_{\Psi, H}(F)=\inf \left\{\beta: \Psi_{H}^{\beta}(F)=0\right\} .
$$


Remark 2.2. Let $\rho_{H}$ be the metric defined on $\mathbb{R}_{+} \times \mathbb{R}^{d}$ by

$$
\rho_{H}((s, x),(t, y))=\max \left\{|s-t|^{H},\|x-y\|_{\infty}\right\} \quad \forall(s, x),(t, y) \in \mathbb{R}_{+} \times \mathbb{R}^{d} .
$$

We define the $\beta$-dimensional Hausdorff content as

$$
\mathcal{H}_{\rho_{H}}^{\beta}(F)=\inf \left\{\sum_{j} \operatorname{diam}\left(U_{j}\right)^{\beta}: F \subseteq \cup_{j} U_{j}\right\}
$$

where $\left\{U_{j}\right\}$ is a countable cover of $F$ by any sets and $\operatorname{diam}\left(U_{j}\right)$ denotes the diameter of a set $U_{j}$ relatively to the metric $\rho_{H}$. For any $F \subseteq \mathbb{R}_{+} \times \mathbb{R}^{d}$, the Hausdorff dimension, in the metric $\rho_{H}$, of $F$ is defined by

$$
\operatorname{dim}_{\rho_{H}}(F)=\inf \left\{\beta: \mathcal{H}_{\rho_{H}}^{\beta}(F)=0\right\}
$$

Since the diameter of the set $\left[a_{j}, a_{j}+\delta_{j}\right] \times\left[b_{j, 1}, b_{j, 1}+\delta_{j}^{H}\right] \times \ldots \times\left[b_{j, d}, b_{j, d}+\delta_{j}^{H}\right]$ is $\delta_{j}^{H}$ then it can be seen without difficulty that for any $\beta>0$ and $F \subseteq \mathbb{R}_{+} \times \mathbb{R}^{d}$ we have

$$
\Psi_{H}^{\beta}(F)=\mathcal{H}_{\rho_{H}}^{\beta / H}(F)
$$

Hence we obtain

$$
\operatorname{dim}_{\Psi, H}(F)=H \times \operatorname{dim}_{\rho_{H}}(F) .
$$

The following proposition relates $\beta$-dimensional capacity to the $H$-parabolic Hausdorff dimension.

Proposition 2.3. Let $F \subset \mathbb{R}_{+} \times \mathbb{R}^{d}$ be a compact set. Then we have

$$
\operatorname{dim}_{\Psi, H}(F)=\sup \left\{\beta: \mathcal{C}_{\rho_{H}, \beta / H}(F)>0\right\}=\inf \left\{\beta: \mathcal{C}_{\rho_{H}, \beta / H}(F)=0\right\},
$$

where $\mathcal{C}_{\rho_{H}, \beta}($.$) is the \beta$-capacity on the metric space $\left(\mathbb{R}_{+} \times \mathbb{R}^{d}, \rho_{H}\right)$ defined by

$$
\mathcal{C}_{\rho_{H}, \beta}(F)=\left[\inf _{\mu \in \mathcal{P}(F)} \int_{\mathbb{R}_{+} \times \mathbb{R}^{d}} \int_{\mathbb{R}_{+} \times \mathbb{R}^{d}} \frac{\mu(d u) \mu(d v)}{\left(\rho_{H}(u, v)\right)^{\beta}}\right]^{-1} .
$$

Here $\mathcal{P}(F)$ is the family of probability measure carried by $F$.

Proof. The proof is the same as in the Euclidean case, see for example Theorem 3.4.2 in [4] or Theorem 4.32 in [15].

The next theorem is the analogue of Frostman's theorem for parabolic Hausdorff dimension. The statement can be found in Taylor and Watson in [19, Lemma 4] and the proof follows along the same lines as the proof of usual Frostman's theorem.

Theorem 2.4. (Frostman's theorem) Let $F$ a Borel set in $\mathbb{R}_{+} \times \mathbb{R}^{d}$. If $\operatorname{dim}_{\Psi, H}(F)>\kappa$, then there exists a Borel probability measure $\mu$ supported on $F$, and a constant $C>0$, such that

$$
\mu\left([a, a+\delta] \times \prod_{j=1}^{d}\left[b_{j}, b_{j}+\delta^{H}\right]\right) \leq C \delta^{\kappa},
$$

for any $\left(a, b_{1}, \cdots, b_{d}\right) \in \mathbb{R}_{+} \times \mathbb{R}^{d}$ and $\delta>0$. 
Now we give a comparison result for the Hausdorff parabolic dimensions with different parameters. We note that it is a generalization of Lemma 5.4 in [3] to the $d$-dimensional case. However, there is a flaw (may be a misprint) in the lower bound of Lemma 5.4. The following proposition corrects it and improves the lower and upper bounds.

Proposition 2.5. Let $F \subset \mathbb{R}_{+} \times \mathbb{R}^{d}$ and $H, H^{\prime} \in(0,1)$ such that $H<H^{\prime}$. Then we have

$\operatorname{dim}_{\Psi, H}(F) \vee\left(\frac{H^{\prime}}{H} \operatorname{dim}_{\Psi, H}(F)+1-\frac{H^{\prime}}{H}\right) \leq \operatorname{dim}_{\Psi, H^{\prime}}(F) \leq\left(\frac{H^{\prime}}{H} \operatorname{dim}_{\Psi, H}(F)\right) \wedge\left(\operatorname{dim}_{\Psi, H}(F)+\left(H^{\prime}-H\right) d\right)$.

An equivalent reformulation is

$$
\frac{H}{H^{\prime}} \operatorname{dim}_{\rho_{H}}(F) \vee\left(\operatorname{dim}_{\rho_{H}}(F)+\frac{1}{H^{\prime}}-\frac{1}{H}\right) \leq \operatorname{dim}_{\rho_{H^{\prime}}}(F) \leq\left(\operatorname{dim}_{\rho_{H}}(F) \wedge\left(d+\frac{H}{H^{\prime}}\left(\operatorname{dim}_{\rho_{H}}(F)-d\right)\right)\right) .
$$

Proof. Firstly let us remark that for the infimum in (2.1) does not change if we consider only $\delta_{j} \leq 1$. Therefore an immediate consequence of the definition is

$$
\operatorname{dim}_{\Psi, H}(F) \leq \operatorname{dim}_{\Psi, H^{\prime}}(F) .
$$

Now let $0<\varepsilon<1$ and $\gamma>\frac{\operatorname{dim}_{\Psi, H^{\prime}}(F)-1}{H^{\prime}}$. Then $\Psi_{H^{\prime}}^{\gamma H^{\prime}+1}(F)=0$, and hence there exists a cover $\left(\left[a_{n}, a_{n}+\delta_{n}\right] \times \prod_{j=1}^{d}\left[b_{n, j}, b_{n, j}+\delta_{n}^{H^{\prime}}\right]\right)_{n \geq 1}$ of the set $F$, such that

$$
\sum_{n \geq 1} \delta_{n}^{\gamma H^{\prime}+1} \leq \varepsilon
$$

It follows from (2.11) that $\delta_{n}<1$ for all $n$. Each interval $\left[a_{n}, a_{n}+\delta_{n}\right]$ can be divided into $\left\lceil\delta_{n}^{1-\frac{H^{\prime}}{H}}\right\rceil$ intervals of length $\delta_{n}^{H^{\prime} / H}$ each. In this way we obtain a new cover $\left(\left[a_{l}^{\prime}, a_{l}^{\prime}+\delta_{l}^{H^{\prime} / H}\right] \times \prod_{j=1}^{d}\left[b_{l, j}^{\prime}, b_{l, j}^{\prime}+\left(\delta_{l}^{H^{\prime} / H}\right)^{H}\right]\right)_{l \geq 1}$ of the set $F$ which satisfies

$$
\sum_{l \geq 1}\left(\delta_{l}^{H^{\prime} / H}\right)^{\gamma H+1} \leq 2 \sum_{n \geq 1} \delta_{n}^{1-\frac{H^{\prime}}{H}}\left(\delta_{n}^{H^{\prime} / H}\right)^{\gamma H+1} \leq 2 \varepsilon
$$

From (2.12) we deduce that $\operatorname{dim}_{\Psi, H}(F) \leq \gamma H+1$, which implies $\frac{\operatorname{dim}_{\Psi, H}(F)-1}{H} \leq \gamma$. Therefore letting $\gamma$ go to $\frac{\operatorname{dim}_{\Psi, H^{\prime}}(F)-1}{H^{\prime}}$ we conclude

$$
\frac{H^{\prime}}{H} \operatorname{dim}_{\Psi, H}(F)+1-\frac{H^{\prime}}{H} \leq \operatorname{dim}_{\Psi, H^{\prime}}(F) .
$$

Combining (2.10) and (2.13), we obtain the lower bound

$$
\operatorname{dim}_{\Psi, H}(F) \vee\left(\frac{H^{\prime}}{H} \operatorname{dim}_{\Psi, H}(F)+1-\frac{H^{\prime}}{H}\right) \leq \operatorname{dim}_{\Psi, H^{\prime}}(F) .
$$


For the second inequality let $\kappa<\operatorname{dim}_{\Psi, H^{\prime}}(F)$. Then by Frostman's theorem 2.4 there exists a probability measure $\mu$ supported on $F$ such that (2.8) is satisfied. Our aim is to show that

$$
\mu\left([a, a+\delta] \times \prod_{j=1}^{d}\left[b_{j}, b_{j}+\delta^{H}\right]\right) \leq C\left(\delta^{\kappa+d\left(H-H^{\prime}\right)} \wedge \delta^{\kappa H / H^{\prime}}\right),
$$

for any $a \in \mathbb{R}_{+}, b_{1}, \ldots, b_{d} \in \mathbb{R}$ and $0<\delta<1$. Note first that since $\delta \leq \delta^{H / H^{\prime}}$ we have

$$
[a, a+\delta] \times \prod_{j=1}^{d}\left[b_{j}, b_{j}+\delta^{H}\right] \subset\left[a, a+\delta^{H / H^{\prime}}\right] \times\left[b_{1}, b_{1}+\left(\delta^{H / H^{\prime}}\right)^{H^{\prime}}\right] \times \ldots \times\left[b_{d}, b_{d}+\left(\delta^{H / H^{\prime}}\right)^{H^{\prime}}\right] .
$$

Using (2.8) we obtain

$$
\mu\left([a, a+\delta] \times \prod_{j=1}^{d}\left[b_{j}, b_{j}+\delta^{H}\right]\right) \leq C \delta^{\kappa H / H^{\prime}}
$$

Now, for any $j=1, \ldots, d$, the interval $\left[b_{j}, b_{j}+\delta^{H}\right]$ can be covered by at most $\delta^{\left(H-H^{\prime}\right)}$ interval of length $\delta^{H^{\prime}}$. Using once again (2.8) we deduce that

$$
\mu\left([a, a+\delta] \times \prod_{j=1}^{d}\left[b_{j}, b_{j}+\delta^{H}\right]\right) \leq C \delta^{\kappa+d\left(H-H^{\prime}\right)} .
$$

Thus the inequality (2.14) is proved. Since, in the metric space $\left(\mathbb{R}_{+} \times \mathbb{R}^{d}, \rho_{H}\right)$, the diameter of the set $[a, a+\delta] \times \prod_{j=1}^{d}\left[b_{j}, b_{j}+\delta^{H}\right]$ is $\delta^{H}$ then the Mass Distribution Principle, (see Theorem 4.19 in [15]), implies that

$$
\operatorname{dim}_{\rho_{H}}(F) \geq \frac{\kappa}{H^{\prime}} \vee \frac{\kappa+d\left(H-H^{\prime}\right)}{H} .
$$

From (2.5) it follows that

$$
\kappa \leq\left(\frac{H^{\prime}}{H} \operatorname{dim}_{\Psi, H}(F)\right) \wedge\left(\operatorname{dim}_{\Psi, H}(F)+d\left(H^{\prime}-H\right)\right) .
$$

Therefore letting $\kappa \uparrow \operatorname{dim}_{\psi, H^{\prime}}(F)$ the assertion (2.9) follows.

Remark 2.6. It is easy to see that

$$
\operatorname{dim}_{\Psi, H}(F) \vee\left(\frac{H^{\prime}}{H} \operatorname{dim}_{\Psi, H}(F)+1-\frac{H^{\prime}}{H}\right)= \begin{cases}\operatorname{dim}_{\Psi, H}(F) & \text { if } \quad \operatorname{dim}_{\Psi, H}(F) \leq 1 \\ \frac{H^{\prime}}{H} \operatorname{dim}_{\Psi, H}(F)+1-\frac{H^{\prime}}{H} & \text { if } \quad \operatorname{dim}_{\Psi, H}(F)>1 .\end{cases}
$$

Hence the lower bound in (2.9) is sharp as long as $\operatorname{dim}_{\Psi, H}(F)>1$.

The following proposition looks at the effect of Hölder continuous maps on the Hausdorff dimension of sets $\operatorname{dim}(A)$ and $\operatorname{dim}_{\Psi, H}\left(G r_{A}(f)\right)$

Proposition 2.7. Let $f:[0,1] \rightarrow \mathbb{R}^{d}$ be a Borel measurable function and $A$ be a Borel subset of $[0,1]$. Then we have

$$
\operatorname{dim}(A) \leq \operatorname{dim}_{\Psi, H}\left(G r_{A}(f)\right)
$$


If in addition the function $f$ is Hölder continuous with exponent $\alpha \leq H$ ( $\alpha$-Hölder continuous), that is

$$
\exists K \geq 0:\|f(x)-f(y)\|_{\infty} \leq K|x-y|^{\alpha}, \forall x, y \in[0,1]
$$

then we have

$$
\operatorname{dim}_{\Psi, H}\left(G r_{A}(f)\right) \leq\left(\frac{H}{\alpha} \operatorname{dim}(A)\right) \wedge(\operatorname{dim}(A)+(H-\alpha) d) .
$$

Especially, when $f$ is $(H-\varepsilon)$-Hölder continuous for all $\varepsilon>0$ then

$$
\operatorname{dim}(A)=\operatorname{dim}_{\Psi, H}\left(G r_{A}(f)\right)
$$

Proof. We begin by proving (2.17). Let $\gamma>\operatorname{dim}_{\Psi, H}\left(G r_{A}(f)\right)$. Since $\Psi_{H}^{\gamma}\left(G r_{A}(f)\right)=0$ then for all $\varepsilon>0$ there exists a cover $\left(\left[a_{l}, a_{l}+\delta_{l}\right] \times \prod_{j=1}^{d}\left[b_{l, j}, b_{l, j}+\delta_{l}^{H}\right]\right)_{l \geq 1}$ of $G r_{A}(f)$ such that $\sum_{l \geq 1} \delta_{l}^{\gamma} \leq \varepsilon$. Consequently, in $\mathbb{R}$ with the absolute-value metric, $\left(\left[a_{l}, a_{l}+\delta_{l}\right]\right)_{l \geq 1}$ is a covering of $A$ such that

$$
\sum_{l \geq 1}\left|\left[a_{l}, a_{l}+\delta_{l}\right]\right|^{\gamma} \leq \varepsilon
$$

Here $\left|\left[a_{l}, a_{l}+\delta_{l}\right]\right|$ is the diameter of the set $\left[a_{l}, a_{l}+\delta_{l}\right]$. Then we deduce that the $\gamma$-dimensional Hausdorff content satisfies

$$
\mathcal{H}^{\gamma}(A):=\inf \left\{\sum_{j}\left|U_{j}\right|^{\gamma}: A \subseteq \cup_{j} U_{j}\right\} \leq \varepsilon .
$$

Thus $\mathcal{H}^{\gamma}(A)=0$ and therefore $\operatorname{dim}(A) \leq \gamma$. Now by making $\gamma \downarrow \operatorname{dim}_{\Psi, H}\left(G r_{A}(f)\right)$ we get the desired inequality.

Now let $\alpha \leq H$ and assume that $f$ is $\alpha$-Hölder continuous. For any $0<\kappa<\operatorname{dim}_{\Psi, H}\left(G r_{A}(f)\right)$, by resorting again to Frostman's theorem there exist a measure $\mu$ on $G r_{A}(f)$ and a constant $C>0$ such that

$$
\mu\left([a, a+\delta] \times \prod_{j=1}^{d}\left[b_{j}, b_{j}+\delta^{H}\right]\right) \leq C \delta^{\kappa}
$$

for any $\left(a, b_{1}, \cdots, b_{d}\right) \in A \times \mathbb{R}^{d}$ and $\delta \in(0,1]$. Let $\nu$ be the measure on $A$ satisfying $\nu=\mu \circ P_{1}^{-1}$ where $P_{1}$ is the projection mapping on $A$, i.e. $P_{1}(s, f(s))=s$. Our aim is to show that

$$
\nu([a, a+\delta])=\mu\left(P_{1}^{-1}([a, a+\delta])\right) \leq C_{1}\left(\delta^{\kappa+d(\alpha-H)} \wedge \delta^{\kappa \alpha / H}\right),
$$

for some constant $C_{1}>0$ and any $\delta \in(0,1]$. Since $f$ is Hölder continuous function with exponent $\alpha$ and constant $K$, then for $s \in[a, a+\delta]$ we have $f_{j}(s) \in\left[f_{j}(a)-K \delta^{\alpha}, f_{j}(a)+K \delta^{\alpha}\right]$. There are two ways to cover

$$
P_{1}^{-1}([a, a+\delta])=\left\{(s, f(s)) \in G r_{A}(f) ; s \in[a, a+\delta]\right\} .
$$

The first one is : for all $j=1, \ldots, d$ we decompose every interval $\left[f_{j}(a)-K \delta^{\alpha}, f_{j}(a)+K \delta^{\alpha}\right]$ into at most $\lceil 2 K\rceil \delta^{(\alpha-H)}$ interval of length $\delta^{H}$, then $P_{1}^{-1}([a, a+\delta])$ is covered by at most $\lceil 2 K\rceil^{d} \delta^{d(\alpha-H)}$ sets of the form

$$
[a, a+\delta] \times \prod_{j=1}^{d}\left[b_{j}, b_{j}+\delta^{H}\right]
$$


With regard to the second way, since $\delta \leq \delta^{\alpha / H}$ then $P_{1}^{-1}([a, a+\delta])$ may be covered by at most $\lceil 2 K\rceil^{d}$ sets of the form

$$
\left[a, a+\delta^{\alpha / H}\right] \times \prod_{j=1}^{d}\left[b_{j}, b_{j}+\delta^{\alpha}\right]
$$

Now using (2.21) we conclude that there exists a constant $C^{\prime}>0$ which depends only on $K$ and $d$, such that

$$
\nu([a, a+\delta])=\sigma\left(P_{1}^{-1}([a, a+\delta])\right) \leq C^{\prime} \delta^{\kappa+d(\alpha-H)},
$$

for the first cover. For the second one we get

$$
\nu([a, a+\delta]) \leq C^{\prime \prime} \delta^{\kappa \alpha / H},
$$

where $C^{\prime \prime}>0$ is a constant depending only on $K$ and $d$. Thus inequality (2.22) is proved. Thanks to the mass distribution principle, see Theorem 4.19 in [15], we obtain

$$
\kappa \leq\left(\frac{H}{\alpha} \operatorname{dim}(A)\right) \wedge(\operatorname{dim}(A)+(H-\alpha) d) .
$$

Therefore letting $\kappa \uparrow \operatorname{dim}_{\Psi, H}\left(G r_{A}(f)\right)$ the assertion (2.18) follows.

Finally it is easy to see from (2.17) and (2.18) that when $f$ is $(H-\varepsilon)$-Hölder continuous for all $\varepsilon>0$ we have $\operatorname{dim}(A)=\operatorname{dim}_{\Psi, H}\left(G r_{A}(f)\right)$.

Remark 2.8. It is worth noting that the inequality (2.18) is also obtained by assuming only that the function $f$ is $(\alpha-\varepsilon)$-Hölder continuous for all $\varepsilon>0$.

A natural question that arises from Proposition 2.7 whether (2.18) is a sharp estimate? The main idea is to use the paths of Hölder continuous stochastic process. Precisely, we have the following result

Theorem 2.9. Let $\alpha \leq H,\left\{B^{\alpha}(t): t \in[0,1]\right\}$ a d-dimensional fractional Brownian motion of Hurst index $\alpha$ and $A \subset[0,1]$ a Borel set. Then we have

$$
\operatorname{dim}_{\Psi, H}\left(G r_{A}\left(B^{\alpha}\right)\right)=\left(\left(\frac{H}{\alpha} \operatorname{dim}(A)\right) \wedge(\operatorname{dim}(A)+d(H-\alpha))\right) \text { a.s. }
$$

Moreover, if $\alpha<H$ we have

$$
\operatorname{dim}_{\Psi, H}\left(G r_{A}\left(B^{\alpha}\right)\right)>\operatorname{dim}(A) .
$$

For the proof we need the following lemma.

Lemma 2.10. There exists a constants $C$ such that for all $t \in(0,1]$ we have

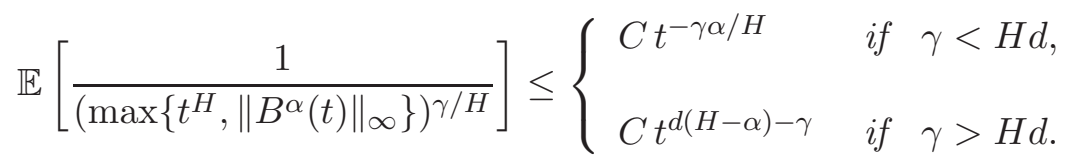

Proof. We first note that the denominator on the left hand side of (2.25) has the same distribution as $\left(\max \left\{t^{H}, t^{\alpha}\|N\|_{\infty}\right\}\right)^{\gamma / H}$, where $N$ is a $d$-dimensional standard normal random variable.

Now assume that $\gamma<H d$. Then we obtain

$$
\mathbb{E}\left[\frac{1}{\left(\max \left(t^{H},\left\|B^{\alpha}(t)\right\|_{\infty}\right)\right)^{\gamma / H}}\right]=\mathbb{E}\left[\frac{1}{\left(\max \left(t^{H}, t^{\alpha}\|N\|_{\infty}\right)\right)^{\gamma / H}}\right] \leq t^{-\alpha \gamma / H} \mathbb{E}\left[\|N\|_{\infty}^{-\gamma / H}\right] .
$$


Since $\gamma<H d$, we have that $\mathbb{E}\left[\|N\|_{\infty}^{-\gamma / H}\right]$ is finite and therefore

$$
\mathbb{E}\left[\frac{1}{\left(\max \left(t^{H},\left\|B^{\alpha}(t)\right\|_{\infty}\right)\right)^{\gamma / H}}\right] \leq C t^{-\alpha \gamma / H}
$$

Next, let $\gamma>H d$. Since $\|y\|_{\infty} \leq\|y\|$ for any $y \in \mathbb{R}^{d}$ it yields

$$
\begin{aligned}
\mathbb{E}\left[\frac{1}{\left(\max \left(t^{H}, t^{\alpha}\|N\|_{\infty}\right)\right)^{\gamma / H}}\right] & =\frac{1}{t^{\gamma}} \mathbb{P}\left[\|N\|_{\infty} \leq t^{H-\alpha}\right]+\frac{1}{t^{\gamma \alpha / H}} \mathbb{E}\left[\frac{1}{\|N\|_{\infty}^{\gamma / H}} \mathbb{1}_{\left\{\|N\|_{\infty}>t^{H-\alpha}\right\}}\right] \\
& \leq C\left(\frac{1}{t^{\gamma}} \int_{\|y\|_{\infty} \leq t^{H-\alpha}} e^{-\|y\|^{2} / 2} d y+\frac{1}{t^{\gamma \alpha / H}} \int_{\|y\|_{\infty}>t^{H-\alpha}} \frac{e^{-\|y\|^{2} / 2}}{\|y\|_{\infty}^{\gamma / H}} d y\right) \\
& \leq C_{1}\left(t^{d(H-\alpha)-\gamma}+t^{-\gamma \alpha / H} \int_{t^{H-\alpha}}^{1} r^{d-1-\gamma / H} d r+t^{-\gamma \alpha / H}\right) \\
& \leq C_{2}\left(t^{d(H-\alpha)-\gamma}+t^{-\gamma \alpha / H}\right) \\
& \leq C_{3} t^{d(H-\alpha)-\gamma},
\end{aligned}
$$

where the constants $C_{j}, j=1,2,3$ depend only on $d, H, \alpha$ and $\gamma$.

Proof of Theorem [2.9. The upper bound of $\operatorname{dim}_{\Psi, H}\left(G r_{A}\left(B^{\alpha}\right)\right)$ follows directly from Proposition 2.7

Now let us prove the lower bound. First, we consider the case $\operatorname{dim}(A) \leq \alpha d$. Let $\gamma<\frac{H}{\alpha} \operatorname{dim}(A) \leq$ $\operatorname{dim}(A)+d(H-\alpha)$. By Theorem 4.32 in [15], there exists a probability measure $\nu$ on $A$ such that

$$
\mathcal{E}_{\gamma \alpha / H}(\nu):=\int_{A} \int_{A} \frac{1}{|t-s|^{\gamma \alpha / H}} \nu(d s) \nu(d t)<\infty .
$$

Let $\widetilde{\mu}$ be the random measure defined by

$$
\widetilde{\mu}(E)=\nu\left\{s:\left(s, B^{\alpha}(s)\right) \in E\right\},
$$

where $E \subset G r_{A}\left(B^{\alpha}\right)$. We will show that

$$
\mathcal{E}_{\rho_{H}, \gamma / H}(\widetilde{\mu}):=\int_{\mathbb{R}_{+} \times \mathbb{R}^{d}} \int_{\mathbb{R}_{+} \times \mathbb{R}^{d}} \frac{\widetilde{\mu}(d u) \widetilde{\mu}(d v)}{\left(\rho_{H}(u, v)\right)^{\gamma / H}}<\infty \text { a.s. }
$$

Taking expectation and using a change of variables and Fubini's theorem we get

$$
\mathbb{E}\left[\mathcal{E}_{\rho_{H}, \gamma / H}(\widetilde{\mu})\right]=\int_{A} \int_{A} \mathbb{E}\left[\frac{1}{\left(\max \left(|s-t|^{H},\left\|B^{\alpha}(t)-B^{\alpha}(s)\right\|_{\infty}\right)\right)^{\gamma / H}}\right] \nu(d s) \nu(d t) .
$$

By recalling the fact that $B^{\alpha}$ has stationary increments we see that the denominator has the same distribution as $\left(\max \left(|s-t|^{H},\left\|B^{\alpha}(|s-t|)\right\|_{\infty}\right)\right)^{\gamma / H}$. It follows that

$$
\mathbb{E}\left[\mathcal{E}_{\rho_{H}, \gamma / H}(\widetilde{\mu})\right]=\int_{A} \int_{A} \mathbb{E}\left[\frac{1}{\left(\max \left(|s-t|^{H},\left\|B^{\alpha}(|s-t|)\right\|_{\infty}\right)\right)^{\gamma / H}}\right] \nu(d s) \nu(d t) .
$$

Since $\gamma<H d$ we deduce from Lemma 2.10 that

$$
\mathbb{E}\left[\mathcal{E}_{\rho_{H}, \gamma / H}(\widetilde{\mu})\right] \leq C \int_{A} \int_{A} \frac{1}{|t-s|^{\gamma \alpha / H}} \nu(d s) \nu(d t)
$$


which is finite from (2.26). Hence $\mathcal{C}_{\rho_{H}, \gamma / H}\left(G r_{A}\left(B^{\alpha}\right)\right)>0$ almost surely and Proposition 2.3 allows that $\operatorname{dim}_{\Psi, H}\left(G r_{A}\left(B^{\alpha}\right)\right) \geq \gamma$ a.s.

Now assume that $\operatorname{dim}(A)>\alpha d$. In this case, we choose $H d<\gamma<\operatorname{dim}(A)+d(H-\alpha)<\frac{H}{\alpha} \operatorname{dim}(A)$. We use the same tools, as in the previous case, to prove that $\mathcal{C}_{\rho_{H}, \gamma / H}\left(G r_{A}\left(B^{\alpha}\right)\right)>0$ a.s via the probability measure $\nu$ satisfying

$$
\mathcal{E}_{\gamma-d(H-\alpha)}(\nu)<\infty .
$$

Letting $\gamma \uparrow\left(\frac{H}{\alpha} \operatorname{dim}(A)\right) \wedge(\operatorname{dim}(A)+d(H-\alpha))$ finishes the proof.

As a consequence of (2.5) and Theorem 2.9, we have the following result

Corollary 2.11. Let $\alpha \leq H,\left\{B^{\alpha}(t): t \in[0,1]\right\}$ a fractional Brownian motion of Hurst index $\alpha$ and $A \subset[0,1]$ a Borel set. Then we have

$$
\operatorname{dim}_{\Psi, H}\left(G r_{A}\left(B^{\alpha}\right)\right)>H d \text { a.s } \Longleftrightarrow \operatorname{dim}_{\rho_{H}}\left(G r_{A}\left(B^{\alpha}\right)\right)>d \text { a.s } \Longleftrightarrow \operatorname{dim}(A)>\alpha d .
$$

\section{Positive Lebesgue measure and non-empty interior of $\left(B^{H}+f\right)(A)$}

Let $Y=(Y(t))_{t \in[0,1]}$ be an $\mathbb{R}^{d}$-valued stochastic process and $\nu$ is a positive measure on $[0,1]$. The occupation measure of the sample path $[0,1] \ni t \longrightarrow Y(t)(w) \in \mathbb{R}^{d}$ is defined by

$$
\nu_{Y}(E):=\nu\{t \in[0,1]: Y(t) \in E\},
$$

where $E \subset \mathbb{R}^{d}$ is a Borel set. We say that $Y$ has an occupation density relative to the Lebesgue measure $\lambda_{d}$ if $\nu_{Y}$ is absolutely continuous with respect to $\lambda_{d}$ almost surely.

Simple modifications of the differentiation's method, see Theorem 21.15 in [7, allow to give necessary and sufficient conditions under which $\nu_{Y}$ has an occupation density relative to the Lebesgue measure $\lambda_{d}$ a.s. Hence we omit the proof. Here is a precise statement:

Proposition 3.1. The following assertions are equivalent:

$$
\begin{aligned}
& \text { 1. } \nu_{Y}<<\lambda_{d} \text { with } \frac{d \nu_{Y}}{d \lambda_{d}}(.) \in L^{2}\left(\lambda_{d} \otimes \mathbb{P}\right) . \\
& \text { 2. } \quad \liminf _{r \downarrow 0} r^{-d} \int_{A} \int_{A} \mathbb{P}\{\|Y(s)-Y(t)\|<r\} d \nu(s) d \nu(t)<\infty .
\end{aligned}
$$

Let $A \subset[0,1]$ and assume that $\eta:=\operatorname{dim}_{\Psi, H}\left(G r_{A}(f)\right)>H d$. It follows from Theorem 2.4 that for $\kappa \in(H d, \eta)$ there exists a Borel probability measure $\mu$ supported on $G r_{A}(f)$ and $C>0$ such that

$$
\mu\left([a, a+\delta] \times \prod_{j=1}^{d}\left[b_{j}, b_{j}+\delta^{H}\right]\right) \leq C \delta^{\kappa},
$$

for all $a \in A, b_{1}, \ldots, b_{n} \in \mathbb{R}^{d}$ and all $\delta \in(0,1]$. Let $\nu$ be the measure defined on $A$ by $\nu=\mu \circ P_{1}^{-1}$. We are now ready to state

Theorem 3.2. Let $\left\{B^{H}(t): t \in[0,1]\right\}$ be a d-dimensional fractional Brownian motion of Hurst index $H \in(0,1)$. Let $f:[0,1] \rightarrow \mathbb{R}^{d}$ be a Borel measurable function and let $A \subset[0,1]$ be a Borel set. If $\eta:=\operatorname{dim}_{\Psi, H}\left(G r_{A}(f)\right)>H d$ then $\lambda_{d}\left(\left(B^{H}+f\right)(A)\right)>0$ almost surely. 
Proof. To achieve this purpose, it is enough to verify the second assertion of Proposition 3.1 for the process $B^{H}+f$. Indeed for $s, t \in A$ and $r>0$, we have

$$
\mathbb{P}\left\{\left\|\left(B^{H}+f\right)(s)-\left(B^{H}+f\right)(t)\right\|<r\right\}=\frac{1}{(2 \pi)^{d / 2}|t-s|^{H d}} \int_{\|y\|<r} \exp \left(-\frac{\|y-f(t)+f(s)\|^{2}}{2|t-s|^{2 H}}\right) d y .
$$

Then using Fubini's theorem we obtain, for any fixed $t \in A$ and $r>0$, that

$$
\begin{aligned}
\int_{A} \mathbb{P}\left\{\left\|\left(B^{H}+f\right)(s)-\left(B^{H}+f\right)(t)\right\|<r\right\} d \nu(s) & =\frac{1}{(2 \pi)^{d / 2}} \int_{\|y\|<r} \int_{A} \frac{1}{|t-s|^{H d}} \exp \left(-\frac{\|y-f(t)+f(s)\|^{2}}{2|t-s|^{2 H}}\right) d \nu(s) d y \\
& \leq C r^{d} \sup _{\|y\|<r} \underbrace{\int_{A} \frac{1}{|t-s|^{H d}} \exp \left(-\frac{\|y-f(t)+f(s)\|^{2}}{2|t-s|^{2 H}}\right) d \nu(s)}_{=I(y,(t, f(t)))} .
\end{aligned}
$$

where $C$ is a positive constant depending only on $d$.

For any fixed $y$ in $\{\|y\|<r\}$ and $(t, f(t)) \in G r_{A}(f)$, we have the following decomposition

$$
I(y,(t, f(t)))=I_{1}(y,(t, f(t)))+I_{2}(y,(t, f(t))),
$$

where

$$
\begin{aligned}
& I_{1}(y,(t, f(t)))=\int_{\left\{s \in A:\|y-f(t)+f(s)\| \leq C_{1}|s-t|^{H} \sqrt{|\log | s-t||}\right\}} \frac{1}{|s-t|^{H d}} \exp \left(-\frac{\|y-f(t)+f(s)\|^{2}}{2|s-t|^{2 H}}\right) d \nu(s) \\
& =\int_{\left\{(s, f(s)) \in G r_{A}(f):\|y-f(t)+f(s)\| \leq C_{1}|s-t|^{H} \sqrt{|\log | s-t||}\right\}} \frac{1}{|s-t|^{H d}} \exp \left(-\frac{\|y-f(t)+f(s)\|^{2}}{2|s-t|^{2 H}}\right) d \mu(s, f(s)) .
\end{aligned}
$$

and

$$
\begin{aligned}
& I_{2}(y,(t, f(t)))=\int_{\left\{s \in A:\|y-f(t)+f(s)\|>C_{1}|s-t|^{H} \sqrt{|\log | s-t||}\right\}} \frac{1}{|s-t|^{H d}} \exp \left(-\frac{\|y-f(t)+f(s)\|^{2}}{2|s-t|^{2 H}}\right) d \nu(s) \\
& =\int_{\left\{(s, f(s)) \in G r_{A}(f):\|y-f(t)+f(s)\|>C_{1}|s-t|^{H} \sqrt{|\log | s-t||}\right\}} \frac{1}{|s-t|^{H d}} \exp \left(-\frac{\|y-f(t)+f(s)\|^{2}}{2|s-t|^{2 H}}\right) d \mu(s, f(s)),
\end{aligned}
$$

where $C_{1}$ is a positive constant which will be chosen later. We first show that

$$
\sup _{(y,(t, f(t))) \in\{\|y\|<r\} \times G r_{A}(f)} I_{1}(y,(t, f(t)))<+\infty .
$$

By the inequality (3.1) we can verify that the measure $\nu$ is no atomic. Then we have

$$
\begin{aligned}
I_{1}(y,(t, f(t))) & \leq \sum_{n=1}^{\infty} 2^{n H d} \mu\left\{\|y-f(t)+f(s)\| \leq C_{1}|s-t|^{H} \sqrt{|\log | s-t||}, 2^{-n}<|s-t| \leq 2^{1-n}\right\} \\
& \leq \sum_{n=1}^{\infty} 2^{n H d} \mu\left\{\|y-f(t)+f(s)\| \leq C_{2} 2^{-n H} \sqrt{n}, 2^{-n}<|s-t| \leq 2^{1-n}\right\}
\end{aligned}
$$


where $C_{2}=C_{1} 2^{H} \sqrt{\log (2)}$. Now, for $n \geq 1$, we set

$$
S_{n}(y,(t, f(t)))=\left\{(s, f(s)) \in G r_{A}(f):\|y-f(t)+f(s)\| \leq C_{2} 2^{-n H} \sqrt{n}, 2^{-n}<|s-t| \leq 2^{1-n}\right\} .
$$

For $(s, f(s)) \in S_{n}(y,(t, f(t)))$ we have $s \in\left[t-2^{1-n}, t+2^{1-n}\right]$ and

$$
f_{j}(s) \in J_{j}=\left[f_{j}(t)-y_{j}-C_{2} 2^{-n H} \sqrt{n}, f_{j}(t)-y_{j}+C_{2} 2^{-n H} \sqrt{n}\right]
$$

for all $j \in\{1, \ldots, d\}$. Since $\left[t-2^{1-n}, t+2^{1-n}\right]$ is covered by 4 intervals of length $2^{-n}$ and every $J_{j}$ is covered by no more than a constant multiple (which depends only on $H$ ) of $\sqrt{n}$ intervals of length $2^{-n H}$, then we can cover $S_{n}(y,(t, f(t)))$ by no more than a constant (which depends only on $d$ and $H$ ) multiple of $n^{d / 2}$ sets of the form $\left[a, a+2^{-n}\right] \times \prod_{j=1}^{d}\left[b_{j}, b_{j}+2^{-n H}\right]$. Applying inequality (3.1) allows to have

$$
\sup _{(y,(t, f(t))) \in\{\|y\|<r\} \times G r_{A}(f)} \mu\left(S_{n}(y,(t, f(t))) \leq C_{3} n^{d / 2} 2^{-\kappa n} .\right.
$$

Therefore we get

$$
\sup _{(y,(t, f(t))) \in\{\|y\|<r\} \times G r_{A}(f)} I_{1}(y,(t, f(t))) \leq C_{4} \sum_{n=1}^{\infty} 2^{-(\kappa-H d) n} n^{d / 2}<\infty,
$$

where $C_{4}$ depends on $d$ and $H$ only. For the second term $I_{2}(y,(t, f(t)))$ we have

$$
\begin{aligned}
I_{2}(y,(t, f(t))) \leq & \sum_{n=1}^{\infty} 2^{n H d} \exp \left(-\frac{C_{1}^{2}}{2}(n-1) \ln 2\right) \times \\
& \mu\left\{\|f(s)-f(t)+y\|>C_{1}|s-t|^{H} \sqrt{|\log | s-t||}, 2^{-n}<|s-t| \leq 2^{1-n}\right\}
\end{aligned}
$$

Thus, for $C_{1}>\sqrt{2 H d}$ we obtain

$$
\sup _{(y,(t, f(t))) \in\{\|y\|<r\} \times G r_{A}(f)} I_{2}(y,(t, f(t))) \leq e^{C_{1}^{2} \ln 2 / 2} \sum_{n=1}^{\infty} 2^{-n\left(C_{1}^{2} / 2-H d\right)}<+\infty .
$$

Now putting all this together yields

$$
\sup _{(y,(t, f(t))) \in\{\|y\|<r\} \times G r_{A}(f)} \int_{A} \frac{1}{|s-t|^{H d}} \exp \left(-\frac{\|y-f(t)+f(s)\|^{2}}{2|s-t|^{2 H}}\right) d \nu(s)<\infty .
$$

Thus we get from (3.2) that

$$
\liminf _{r \downarrow 0} r^{-d} \int_{A} \int_{A} \mathbb{P}\left\{\left\|\left(B^{H}+f\right)(s)-\left(B^{H}+f\right)(t)\right\|<r\right\} d \nu(s) d \nu(t)<\infty .
$$

We can therefore state from Proposition 3.1 that the occupation measure $\nu_{B^{H}+f}$ is absolutely continuous with respect to the Lebesgue measure $\lambda_{d}$ a.s. Hence $\lambda_{d}\left(B^{H}+f\right)(A)>0$ a.s.

Remark 3.3. In connection with the existence of the occupation density let us mention that another, often easier to apply, criterion due to Berman tells us that, $\nu_{Y}$ has a density $\frac{d \nu_{Y}}{d \lambda_{d}}(.) \in L^{2}\left(\lambda_{d} \otimes \mathbb{P}\right)$ if and only if

$$
\int_{\mathbb{R}^{d}} \int_{A} \int_{A} \mathbb{E}\left(e^{i\langle\theta,(Y(s)-Y(t)\rangle}\right) d \nu(s) d \nu(t) d \theta<\infty
$$


However, we were unable to apply it to prove the absolute continuity of the occupation measure $\nu_{B^{H}}+f$ with respect to the Lebesgue measure $\lambda_{d}$ a.s. The difficulty stems from the lack of informations on the measure $\nu$. Indeed, a simple calculation using characteristic function of Gaussian random vector yields

$$
\mathbb{E}\left(e^{i\left\langle\theta,\left(B^{H}+f\right)(s)-\left(B^{H}+f\right)(t)\right\rangle}\right)=e^{i\langle\theta, f(s)-f(t)\rangle} \exp \left(-\frac{|s-t|^{2 H}\|\theta\|^{2}}{2}\right) .
$$

Now integrating the modulus we have

$$
\int_{\mathbb{R}^{d}}\left|\mathbb{E}\left(e^{i\left\langle\theta,\left(B^{H}+f\right)(s)-\left(B^{H}+f\right)(t)\right\rangle}\right)\right| d \theta=\frac{(2 \pi)^{d / 2}}{|s-t|^{H d}} .
$$

However we are unable to ensure finiteness of the integral,

$$
\int_{A} \int_{A} \frac{(2 \pi)^{d / 2}}{|s-t|^{H d}} d \nu(s) d \nu(t)
$$

Hence one cannot use Fubini's theorem which make the cited criterion unworkable.

According to the conclusion of Theorem 3.2, it may be expected that the interior of $\left(B^{H}+f\right)(A)$ is not empty. Recall that Kahane (Theorems 1 and 2 p. 267 in [10]) has shown that, for any compact subset $A \subset[0,1], B^{H}(A)$ is a Salem set almost surely if $\operatorname{dim}(A)<H d$ and $B^{H}(A)$ has a non-empty interior almost surely if $\operatorname{dim}(A)>H d$. That's what prompted us to consider the case $H d<\operatorname{dim}(A)$. Let us note that in the light of (2.17), the condition $H d<\operatorname{dim}(A)$ leads to $\operatorname{dim}_{\Psi, H}\left(G r_{A}(f)\right)>H d$ and therefore $\lambda_{d}\left(\left(B^{H}+f\right)(A)\right)>0$ almost surely. Our aim is to prove that, under this condition, the set $\left(B^{H}+f\right)(A)$ not only has a positive measure but also a non-void interior. A key ingredient is the continuity of the occupation density over $A$. Obtaining this continuity we will make use of the following.

It follows from Lemma 7.1 of [17] that, for any $H \in(0,1)$, the real-valued fractional Brownian motion $B_{0}^{H}$ has the following important property of strong local nondeterminism: There exists a constant $0<C<\infty$ such that for all integers $n \geq 1$ and all $t_{1}, \ldots, t_{n}, t \in[0,1]$, we have

$$
\operatorname{Var}\left(B_{0}^{H}(t) / B_{0}^{H}\left(t_{1}\right), \ldots, B_{0}^{H}\left(t_{n}\right)\right) \geq C \min _{0 \leq j \leq n}\left|t-t_{j}\right|^{2 H},
$$

where $\operatorname{Var}\left(B_{0}^{H}(t) / B_{0}^{H}\left(t_{1}\right), \ldots, B_{0}^{H}\left(t_{n}\right)\right)$ denotes the conditional variance of $B_{0}^{H}(t)$ given $B_{0}^{H}\left(t_{1}\right), \ldots, B_{0}^{H}\left(t_{n}\right)$ and $t_{0}=0$. The following elementary formula allows to estimate the determinant of the covariance matrix of the Gaussian random vector $\left(Z_{1}, \ldots, Z_{n}\right)$

$$
\operatorname{det} \operatorname{Cov}\left(Z_{1}, \ldots, Z_{n}\right)=\operatorname{Var}\left(Z_{1}\right) \prod_{k=2}^{n} \operatorname{Var}\left(Z_{k} / Z_{1}, \ldots, Z_{k-1}\right) .
$$

For the vector $\left(B_{0}^{H}\left(t_{1}\right), \ldots, B_{0}^{H}\left(t_{n}\right)\right)$ one obtains

$$
\operatorname{det} \operatorname{Cov}\left(B_{0}^{H}\left(t_{j}\right), 1 \leq j \leq p\right) \geq \prod_{j=1}^{p}\left(\min \left\{\left|t_{j}-t_{i}\right|^{2 H}, 0 \leq i \leq j-1\right\}\right) .
$$

We need also the following lemma which is due to Cuzick and DuPreez [5]

Lemma 3.4. Let $Z_{1}, \ldots, Z_{n}$ be linearly-independent centered Gaussian variables. If $g: \mathbb{R} \longrightarrow \mathbb{R}$ is a Borel measurable function such that

$$
\int_{-\infty}^{\infty} g(v) e^{-\varepsilon v^{2}} d v<\infty
$$


for all $\varepsilon>0$. Then

$$
\begin{aligned}
\int_{\mathbb{R}^{n}} g\left(v_{1}\right) & \exp \left(-\frac{1}{2} \operatorname{Var}\left(\sum_{j=1}^{n} v_{j} Z_{j}\right)\right) d v_{1} \ldots d v_{n} \\
= & \frac{(2 \pi)^{(n-1) / 2}}{\left(\operatorname{det} \operatorname{Cov}\left(Z_{1}, \ldots, Z_{n}\right)\right)^{1 / 2}} \int_{-\infty}^{\infty} g\left(\frac{v}{\sigma_{1}}\right) e^{-v^{2}} d v
\end{aligned}
$$

where $\sigma_{1}^{2}=\operatorname{Var}\left(Z_{1} \mid Z_{2} \ldots Z_{n}\right)$ is the conditional variance of $Z_{1}$ given $Z_{2}, \ldots, Z_{n}$.

The next result, using the above-mentioned preliminaries, strengthens the result of Theorem 3.2 since it shows that $\left(B^{H}+f\right)(A)$ has a non-empty interior.

Theorem 3.5. Let $\left\{B^{H}(t): t \in[0,1]\right\}$ be a d-dimensional fractional Brownian motion of Hurst index $H \in(0,1)$. Let $f:[0,1] \rightarrow \mathbb{R}^{d}$ be a continuous function and let $A \subset[0,1]$ be a closed set. Assume that $\operatorname{dim}(A)>H d$. Then we have $\left(B^{H}+f\right)(A)$ has a non-empty interior almost surely.

Proof. The usual Frostman's theorem ensure that for all $H d<\kappa<\operatorname{dim}(A)$ there is a constant $C>0$ and a Borel probability measure $\nu$ on $A$ such that

$$
\tau([a, a+\delta]) \leq C \delta^{\kappa}
$$

for all $a \in A$ and $0<\delta<1$. it is easily verified that

$$
\int_{\mathbb{R}^{d}} \int_{A} \int_{A} \mathbb{E}\left(e^{i\left\langle\theta,\left(B^{H}+f\right)(s)-\left(B^{H}+f\right)(t)\right\rangle}\right) d \tau(s) d \tau(t) d \theta \leq(2 \pi)^{d / 2} \int_{A} \int_{A} \frac{d \tau(s) d \tau(t)}{|t-s|^{H d}},
$$

which is finite by 3.7 since $\kappa>H d$. Hence the condition 3.5 for the process $B^{H}+f$ and the probability measure $\tau$ is checked. Consequently, the occupation measure $\tau_{B^{H}+f}$ has an occupation density relative to the Lebesgue measure $\lambda_{d}$ a.s denoted by $\vartheta$. Hence, in order to prove our theorem, it is sufficient to prove that $\vartheta$ has a continuous version.

First of all, let us note that the arguments used to show (25.7) in [7] can be modified to see that, for all $x, y \in \mathbb{R}^{d}$ and for all even integers $p \geq 2$, we have

$$
\begin{aligned}
\mathbb{E}(\vartheta(x)-\vartheta(y))^{p}= & (2 \pi)^{-p d} \int_{A^{p}} \int_{\mathbb{R}^{p d}} \prod_{j=1}^{p}\left(\exp \left(i\left\langle x, \xi_{j}\right\rangle\right)-\exp \left(i\left\langle y, \xi_{j}\right\rangle\right)\right) \\
& \mathbb{E} \exp \left(i \sum_{j=1}^{p}\left\langle\xi_{j},\left(B^{H}+f\right)\left(t_{j}\right)\right\rangle\right) d \xi_{1} \ldots d \xi_{p} d \tau\left(t_{1}\right) \ldots d \tau\left(t_{p}\right) .
\end{aligned}
$$

Let $p \geq 2$ be a fixed even integer and $0<\gamma<1$ whose value will be determined later. Using the facts that

$$
\left|e^{i u}-1\right| \leq 2^{1-\gamma}|u|^{\gamma}, \forall u \in \mathbb{R}
$$

and $|a+b|^{\gamma} \leq|a|^{\gamma}+|b|^{\gamma}$, we have

$$
\prod_{j=1}^{p}\left|\exp \left(i\left\langle x, \xi_{j}\right\rangle\right)-\exp \left(i\left\langle y, \xi_{j}\right\rangle\right)\right| \leq 2^{(1-\gamma) p}\|x-y\|^{\gamma p} \sum \prod_{j=1}^{p}\left|\xi_{j, k_{j}}\right|^{\gamma}
$$


where the summation $\sum$ is taken over all $\left(k_{1}, \ldots, k_{p}\right) \in\{1, \ldots, d\}^{p}$. It follows that

$$
\begin{aligned}
\mathbb{E}(\vartheta(x)-\vartheta(y))^{p} \leq & (2 \pi)^{-p d} 2^{(1-\gamma) p}\|x-y\|^{\gamma p} \sum \int_{A^{p}} \int_{\mathbb{R}^{p d}} \prod_{j=1}^{p}\left|\xi_{j, k_{j}}\right|^{\gamma} \\
& \exp \left(-\frac{1}{2} \operatorname{Var}\left(\sum_{j=1}^{p}\left\langle\xi_{j}, B^{H}\left(t_{j}\right)\right\rangle\right)\right) d \xi_{1} \ldots d \xi_{p} d \tau\left(t_{1}\right) \ldots d \tau\left(t_{p}\right) .
\end{aligned}
$$

Now the generalized Hölder's inequality leads to

$$
\begin{aligned}
\mathbb{E}(\vartheta(x)-\vartheta(y))^{p} \leq & (2 \pi)^{-p d} 2^{(1-\gamma) p}\|x-y\|^{\gamma p} \sum \int_{A^{p}} \prod_{j=1}^{p} \\
& {\left[\int_{\mathbb{R}^{p d}}\left|\xi_{j, k_{j}}\right|^{p \gamma} \exp \left(-\frac{1}{2} \operatorname{Var}\left(\sum_{j=1}^{p}\left\langle\xi_{j}, B^{H}\left(t_{j}\right)\right\rangle\right)\right) d \xi_{1} \ldots d \xi_{p}\right]^{1 / p} d \tau\left(t_{1}\right) \ldots d \tau\left(t_{p}\right) . }
\end{aligned}
$$

Fix a sequence $\left(k_{1}, \ldots, k_{p}\right) \in\{1, \ldots, d\}^{p}, 0<t_{1}<\ldots<t_{p}$, and consider

$$
J=\prod_{j=1}^{p}\left[\int_{\mathbb{R}^{p d}}\left|\xi_{j, k_{j}}\right|^{p \gamma} \exp \left(-\frac{1}{2} \operatorname{Var}\left(\sum_{j=1}^{p}\left\langle\xi_{j}, B^{H}\left(t_{j}\right)\right\rangle\right)\right) d \xi_{1} \ldots d \xi_{p}\right]^{1 / p}
$$

Note that $\left\{B_{l}^{H}, 1 \leq l \leq d\right\}$ are independent copies of $B_{0}^{H}$. The strong local nondeterminism of the fractional Brownian motion $B_{0}^{H}$ and (3.6) , ensure that the random variables $\left\{B_{l}^{H}\left(t_{j}\right), 1 \leq l \leq d ; 1 \leq j \leq p\right\}$ are linearly independent. Hence by applying Lemma 3.4, we derive that $J$ is bounded by

$$
\begin{aligned}
J & \leq \frac{(2 \pi)^{(p d-1) / 2}}{\left[\operatorname{det} \operatorname{Cov}\left(B_{l}^{H}\left(t_{j}\right), 0 \leq l \leq d, 1 \leq j \leq p\right)\right]^{1 / 2}} \int_{\mathbb{R}}|r|^{p \gamma} e^{-r^{2} / 2} d r \prod_{j=1}^{p} \frac{1}{\sigma_{j}^{\gamma}} \\
& \leq \frac{(d K)^{p}(p !)^{\gamma}}{\left[\operatorname{det} \operatorname{Cov}\left(B_{0}^{H}\left(t_{j}\right), 1 \leq j \leq p\right)\right]^{d / 2}} \prod_{j=1}^{p} \frac{1}{\sigma_{j}^{\gamma}}
\end{aligned}
$$

where $\sigma_{j}^{2}$ is the conditional variance of $B_{k_{j}}^{H}\left(t_{j}\right)$ given $B_{l}^{H}\left(t_{i}\right)\left(l \neq k_{j}\right.$ or $\left.l=k_{j}, i \neq j\right)$ and the last inequality follows from Stirling's formula. Combining (3.8) with the well-known facts, see for example the proof of Lemma 2.5 in [20], that

$$
\prod_{j=1}^{p} \frac{1}{\sigma_{j}^{\gamma}} \leq \frac{K^{p}}{\left[\operatorname{det} \operatorname{Cov}\left(B_{0}^{H}\left(t_{j}\right), 1 \leq j \leq p\right)\right]^{\gamma}} \leq \frac{K^{p}}{\prod_{j=1}^{p}\left(\min \left\{\left|t_{j}-t_{i}\right|^{2 H \gamma}, 0 \leq i \leq j-1\right\}\right)}
$$

we conclude

$$
J \leq \frac{\left(d K^{2}\right)^{p}(p !)^{\delta}}{\prod_{j=1}^{p}\left(\min \left\{\left|t_{j}-t_{i}\right|^{H(d+2 \gamma)}, 0 \leq i \leq j-1\right\}\right)} .
$$


Now, we can prove that

$$
\int_{A^{p}} \frac{d \tau\left(t_{1}\right) \ldots d \tau\left(t_{p}\right)}{\prod_{j=1}^{p}\left(\min \left\{\left|t_{j}-t_{i}\right|, 0 \leq i \leq j-1\right\}\right)^{H(d+2 \gamma)}} \leq p !\left(\sup _{s \in[0,1]} \int_{A} \frac{d \tau(t)}{|t-s|^{H(d+2 \gamma)}}\right)^{p} .
$$

First, for all $j \in\{1, \ldots, p\}$ we splite $A$ into the regions $A_{i, j}:=\left\{t \in A \cap I,\left|t-t_{i}\right|=\min \left\{\left|t-t_{l}\right|, 0 \leq l \leq j-1\right\}\right\}$ for all $i \in\{0, \ldots, j-1\}$, and $t_{0}=0$. Then, for all $j \in\{1, \ldots, p\}$ we have

$$
\int_{A} \frac{d \nu(t)}{\left(\min \left\{\left|t-t_{i}\right|, 0 \leq i \leq j-1\right\}\right)^{H(d+2 \gamma)}}=\sum_{i=0}^{j-1} \int_{A_{i, j}} \frac{d \nu(t)}{\left|t-t_{i}\right|^{H(d+2 \gamma)}} \leq j \sup _{s \in[0,1]} \int_{A} \frac{d \nu(t)}{|t-s|^{H(d+2 \gamma)}}
$$

Hence (3.11) follows by iterating the above estimation, and by using (3.7), if we take

$$
0<\gamma<\frac{1}{2}(\kappa / H-d)
$$

the right hand side in (3.11) is finite. Putting all the above facts together, we arrive at

$$
\mathbb{E}(\vartheta(x)-\vartheta(y))^{p} \leq C_{p, \gamma}\|x-y\|^{\gamma p},
$$

where $C_{p, \gamma}$ is a constant depends only on $p$ and $\gamma$. Hence we can apply the Kolmogorov's continuity theorem (Theorem 2.3.1 p.158 in [12]) to get a continuous version on $\mathbb{R}^{d}$ of $\vartheta($.$) . Since A$ is a compact subset of $[0,1]$ (closed subset of $[0,1])$ and $\left(B^{H}+f\right)$ is continuous then $\left(B^{H}+f\right)(A)$ is a compact set in $\mathbb{R}^{d}$. Taking into account that $\vartheta$ has a version which is continuous in $x$ then it follows from Pitt [17, p. 324] or Geman and Horowitz [7, p. 12] that $\{x: \vartheta(x)>0\}$ is open, non-empty and contained in $\left(B^{H}+f\right)(A)$ almost surely. This ends the proof.

Remark 3.6. It is worth noting that the condition $\operatorname{dim}_{\Psi, H}\left(G r_{A}(f)\right)>H d$ in Theorem 3.2 is a weaker than $\operatorname{dim}(A)>H d$ in Theorem 3.5. Indeed let us take $A$ the classical middle thirds Cantor set which Hausdorff dimension $\operatorname{dim}(A)=\frac{\ln (2)}{\ln (3)}$. For $0<\alpha d<\frac{\ln (2)}{\ln (3)}<H d$, we have from (2.23) that $\operatorname{dim}_{\Psi, H}\left(\operatorname{Gr}_{A}\left(B^{\alpha}\right)\right)>$ $H d$. Then by choosing $f$ as a sample function of $B^{\alpha}$ we obtain

$$
\operatorname{dim}(A)<H d<\operatorname{dim}_{\Psi, H}\left(G r_{A}(f)\right) .
$$

Moreover, as can be seen from the proofs of Theorems 3.2 and 3.5, we were able to prove the existence of an occupation density through the conditions $\operatorname{dim}_{\Psi, H}\left(G r_{A}(f)\right)>H d$ and $\operatorname{dim}(A)>H d$ using respectively two different measures namely $\nu$ and $\tau$. It should be noted that these two measures are constructed by Frostman's lemma. But it was simpler and more practical to work with $\tau$ thanks to the advantageous properties that presents, which are consequences of the condition $\operatorname{dim}(A)>H d$, and who have played a key role in the fact that $\left(B^{H}+f\right)(A)$ has a non-empty interior. We are therefore naturally led to ask the following question that we have not solved: can we establish the result of Theorem 3.5 by assuming only that $\operatorname{dim}_{\Psi, H}\left(G r_{A}(f)\right)>H d$ ?

\section{Hölder drifts for which $\left(B^{H}+f\right)(A)$ has a non-empty interior a.s.}

The aim of this section is to consider the case $\operatorname{dim}(A) \leq H d$. Precisely, we seek to construct Hölder functions for which the range $\left(B^{H}+f\right)(A)$ has non-empty interior. Let us make this precise in the following statement. 
Theorem 4.1. Let $\left\{B^{H}(t): t \in[0,1]\right\}$ be a d-dimensional fractional Brownian motion of Hurst index $H \in(0,1)$. Let $A \subset[0,1]$ a closed set such that $0<\operatorname{dim}(A) \leq H d$. Then for all $\alpha \in(0, \operatorname{dim}(A) / d)$ there exists a $\alpha$-Hölder continuous function $f:[0,1] \rightarrow \mathbb{R}^{d}$ for which the range $\left(B^{H}+f\right)(A)$ has a non-empty interior a.s.

The remainder of this section is devoted to the proof of this theorem. To that end, we will make some preparations. Let $\left(\Omega^{\prime}, \mathcal{F}^{\prime}, \mathbb{P}^{\prime}\right)$ be another probability space and $\left\{B^{\alpha^{\prime}}(t): t \in[0,1]\right\}$ be a fractional Brownian motion with Hurst parameter $\alpha^{\prime} \in(0, \operatorname{dim}(A) / d)$ defined on it. Let us consider the $d$-dimensional centred Gaussian process $Z$ defined on $\left(\Omega \times \Omega^{\prime}, \mathcal{F} \times \mathcal{F}^{\prime}, \mathbb{P} \otimes \mathbb{P}^{\prime}\right)$ by

$$
Z\left(t,\left(\omega, \omega^{\prime}\right)\right)=B^{H}(t, \omega)+B^{\alpha^{\prime}}\left(t, \omega^{\prime}\right) \text { for all } t \in[0,1] \text { and }\left(\omega, \omega^{\prime}\right) \in \Omega \times \Omega^{\prime} .
$$

It is easy to see that $Z=\left(Z_{1}, \ldots, Z_{d}\right)$ where $Z_{i}$ are independent copies of the real-valued Gaussian process $Z_{0}=B_{0}^{H}+B_{0}^{\alpha^{\prime}}$ with the covariance function given by

$\widetilde{\mathbb{E}}\left(Z_{0}(s) Z_{0}(t)\right)=\mathbb{E}\left(B_{0}^{H}(s) B_{0}^{H}(t)\right)+\mathbb{E}^{\prime}\left(B_{0}^{\alpha^{\prime}}(s) B_{0}^{\alpha^{\prime}}(t)\right)=\frac{1}{2}\left(s^{2 H}+t^{2 H}-|t-s|^{2 H}\right)+\frac{1}{2}\left(s^{2 \alpha^{\prime}}+t^{2 \alpha^{\prime}}-|t-s|^{2 \alpha^{\prime}}\right)$,

where $\widetilde{\mathbb{E}}$ and $\mathbb{E}^{\prime}$ denote the expectation under the probability $\widetilde{\mathbb{P}}=\mathbb{P} \otimes \mathbb{P}^{\prime}$ and $\mathbb{P}^{\prime}$ respectively.

The following proposition is about the local nondeterminism property of the process $Z$. Let $I \subset(0,1]$ be a closed interval, then we have

Proposition 4.2. The real-valued process $\left\{Z_{0} ; t \geq 0\right\}$ satisfy the following

1. For any $s, t \in I$, we have

$$
|s-t|^{2 \alpha^{\prime}} \leq \widetilde{\mathbb{E}}\left(Z_{0}(t)-Z_{0}(s)\right)^{2} \leq 2|s-t|^{2 \alpha^{\prime}} .
$$

2. There exists a positive constant $C$ depending on $\alpha^{\prime}, H$ and $I$ only, such that

$$
\operatorname{Var}\left(Z_{0}(u) \mid Z_{0}\left(t_{1}\right), \ldots, Z_{0}\left(t_{n}\right)\right) \geq C\left[\min _{0 \leq k \leq n}\left|u-t_{k}\right|^{2 \alpha^{\prime}}+\min _{0 \leq k \leq n}\left|u-t_{k}\right|^{2 H}\right]
$$

for all integers $n \geq 1$, all $u, t_{1}, \ldots, t_{n} \in I$ and $t_{0}=0$.

3. There exists a positive constant $C$ depending on $\alpha^{\prime}, H$ and $I$ only, such that for any $t \in I$ and any $0<r \leq t$,

$$
\operatorname{Var}\left(Z_{0}(t)\left|Z_{0}(s),\right| s-t \mid \geq r\right) \geq C r^{2 \alpha^{\prime}} .
$$

Proof. 1. For any $s, t \in I$, we have

$$
\begin{aligned}
\widetilde{\mathbb{E}}\left(Z_{0}(t)-Z_{0}(s)\right)^{2} & =\mathbb{E}\left(B_{0}^{H}(t)-B_{0}^{H}(s)\right)^{2}+\mathbb{E}^{\prime}\left(B_{0}^{\alpha^{\prime}}(t)-B_{0}^{\alpha^{\prime}}(s)\right)^{2} \\
& =|t-s|^{2 H}+|t-s|^{2 \alpha^{\prime}}
\end{aligned}
$$

Since $\alpha \leq H$ it follows that

$$
|t-s|^{2 \alpha^{\prime}} \leq \widetilde{\mathbb{E}}\left(Z_{0}(t)-Z_{0}(s)\right)^{2} \leq 2|t-s|^{2 \alpha^{\prime}} .
$$


2. By definition we can write

$$
\begin{aligned}
\operatorname{Var}\left(Z_{0}(u) \mid Z_{0}\left(t_{1}\right), \ldots, Z_{0}\left(t_{n}\right)\right)= & \inf _{a_{j} \in \mathbb{R}, 1 \leq j \leq n} \widetilde{\mathbb{E}}\left(Z_{0}(u)-\sum_{j=1}^{p} a_{j} Z_{0}\left(t_{j}\right)\right)^{2} \\
= & \inf _{a_{j} \in \mathbb{R}, 1 \leq j \leq n}\left[\mathbb{E}\left(B_{0}^{H}(u)-\sum_{j=1}^{p} a_{j} B_{0}^{H}\left(t_{j}\right)\right)^{2}+\mathbb{E}^{\prime}\left(B_{0}^{\alpha^{\prime}}(u)-\sum_{j=1}^{p} a_{j} B_{0}^{\alpha^{\prime}}\left(t_{j}\right)\right)^{2}\right] \\
\geq & \inf _{a_{j} \in \mathbb{R}, 1 \leq j \leq n} \mathbb{E}\left(B_{0}^{H}(u)-\sum_{j=1}^{p} a_{j} B_{0}^{H}\left(t_{j}\right)\right)^{2} \\
& \inf _{b_{j} \in \mathbb{R}, 1 \leq j \leq n} \mathbb{E}^{\prime}\left(B_{0}^{\alpha^{\prime}}(u)-\sum_{j=1}^{p} b_{j} B_{0}^{\alpha^{\prime}}\left(t_{j}\right)\right)^{2} \\
= & \operatorname{Var}_{\operatorname{lar}\left(B_{0}^{H}(u) \mid B_{0}^{H}\left(t_{1}\right), \ldots, B_{0}^{H}\left(t_{n}\right)\right)+\operatorname{Var}\left(B_{0}^{\alpha^{\prime}}(u) \mid B_{0}^{\alpha^{\prime}}\left(t_{1}\right), \ldots, B_{0}^{\alpha^{\prime}}\left(t_{n}\right)\right)}
\end{aligned}
$$

By the property of strong local nondeterminism of fractional Brownian motion there exists a positive constant $C$ depending on $\alpha, H$ and $I$ only, such that for all integers $n \geq 1$, all $u, t_{1}, \ldots, t_{n} \in I$,

$$
\operatorname{Var}\left(Z_{0}(u) \mid Z_{0}\left(t_{1}\right), \ldots, Z_{0}\left(t_{n}\right)\right) \geq C\left[\min _{0 \leq k \leq n}\left|u-t_{k}\right|^{2 \alpha^{\prime}}+\min _{0 \leq k \leq n}\left|u-t_{k}\right|^{2 H}\right] .
$$

3. We note that, in the Hilbert space setting, the conditional variance in (4.4) is the squared distance of $Z_{0}(t)$ from the linear subspace spanned by $\left\{Z_{0}(s),|s-t| \geq r\right\}$ in $L^{2}(\widetilde{\mathbb{P}})$. Hence it is sufficient to show that there exists a positive constant $C$ depending on $\alpha^{\prime}$ and $H$ only, such that for all integers $n \geq 1, a_{j} \in \mathbb{R}$ and $s_{j} \in I$ satisfying $\left|s_{j}-t\right| \geq r,(j=1, \cdots, n)$,

$$
\widetilde{\mathbb{E}}\left(Z_{0}(t)-\sum_{j=1}^{p} a_{j} Z_{0}\left(s_{j}\right)\right)^{2} \geq C r^{2 \alpha^{\prime}} .
$$

What follows from (4.3). Thus the proof is ended.

The following is a direct consequence of Corollary 3.3 in [18].

Proposition 4.3. $Z(A)$ has $\mathbb{P} \otimes \mathbb{P}^{\prime}$-a.s. non-empty interior.

Proof of Theorem 4.1. Let $\alpha^{\prime} \in(\alpha, \operatorname{dim}(A) / d)$. It follows from Proposition 4.3 that $Z(A)=\left(B^{H}+\right.$ $\left.B^{\alpha^{\prime}}\right)(A)$ has $\mathbb{P} \otimes \mathbb{P}^{\prime}$-a.s. a non-void interior. Therefore, there exists a $\mathbb{P}^{\prime}$-negligible set $N^{\prime}$ such that, for any $\omega^{\prime} \in N^{\prime c}$, there exists a $\mathbb{P}$-negligible set $N$ with for any $\omega \in N^{c}$ the set $\left(B^{H}(\omega)+B^{\alpha^{\prime}}\left(\omega^{\prime}\right)\right)(A)$ has a non-void interior. It is a well known fact that almost all trajectories of $B^{\alpha^{\prime}}$ are $\alpha$-Hölder continuous, and hence the desired drift can be chosen as a sample function of $B^{\alpha^{\prime}}$.

\section{References}

[1] R. J. Adler. The Geometry of Random Fields. Wiley, New York, 1981. 
[2] T. Antunović, Y. Peres and B. Vermesi. Brownian motion with variable drift can be space filling. Proc. Amer. Math. Soc. 139, no. 9 (2011), 3359-3373.

[3] P. Balança. Some sample path properties of multifractional Brownian motion. Stochastic Process. Appl. 125, no. 10 (2015), 3823-3850.

[4] C. J. Bishop and Y. Peres. Fractals in probability and analysis. Cambridge Studies in Advanced Mathematics, 162. Cambridge University Press, Cambridge, 2017.

[5] J. Cuzick and J. P. DuPreez. Joint continuity of Gaussian local times. Ann. Probab. 10, no. 3 (1982), 810-817.

[6] K. J. Falconer. Fractal geometry-mathematical foundations and applications. John Wiley Sons, Chichester, 1990.

[7] D. Geman and J. Horowitz. Occupation densities. Ann. Probab. 8, no. 1 (1980), 1-67.

[8] D. Geman, J. Horowitz and J. Rosen. A local time analysis of intersections of Brownian motion in the plane. Ann. Probab. 12 (1984), 86-107.

[9] S. E. Graversen. Polar-functions for Brownian motion. Z. Wahrsch. Verw. Gebiete 61, no. 2 (1982), 261-270.

[10] J. P. Kahane. Some random series of functions, Second edition. Cambridge Studies in Advanced Mathematics, 5. Cambridge University Press, Cambridge, 1985.

[11] R. Kaufman. Une propriété métrique du mouvement brownien. C. R. Acad. Sci. Paris Sér. A-B 268 (1969), A727-A728.

[12] D. Khoshnevisan. Multiparameter processes. An introduction to random fields. Springer-Verlag, New York, 2002.

[13] D. Khoshnevisan and Y. Xiao. Brownian motion and thermal capacity. Ann. Probab. 43, no. 1 (2015), 405-434.

[14] J. F. Le Gall. Sur les fonctions polaires pour le mouvement brownien. Séminaire de Probabilités, XXII, 186-189, Lecture Notes in Math., 1321, Springer, Berlin, 1988.

[15] P. Mörters and Y. Peres. Brownian motion. Cambridge Series in Statistical and Probabilistic Mathematics, 30. Cambridge University Press, Cambridge, 2010.

[16] Y. Peres and P. Sousi. Dimension of fractional Brownian motion with variable drift. Probab. Theory Related Fields 165, no. 3-4 (2016), 771-794.

[17] L. D. Pitt. Local times for Gaussian vector fields. Indiana Univ. Math. J. 27, no. 2 (1978), 309-330.

[18] R. Shieh. and Y. Xiao. Images of Gaussian random fields: Salem sets and interior points. Studia Math. 176, no. 1 (2006), 37-60.

[19] S. J. Taylor and N. A. Watson. A Hausdorff measure classification of polar sets for the heat equation. Math. Proc. Cambridge Philos. Soc. 97, no. 2 (1985), 325-344.

[20] Y. Xiao. Hölder conditions for the local times and the Hausdorff measure of the level sets of Gaussian random fields. Probab. Theory Related Fields 109, no. 1 (1997), 129-157 